\title{
Survival Benefit of Neoadjuvant Chemotherapy with S-1 Plus Docetaxel for Locally Advanced Gastric Cancer: A Propensity Score-Matched Analysis
}

\author{
Masayuki Kano, MD, PhD, Koichi Hayano, MD, PhD, Hideki Hayashi, MD, PhD, Naoyuki Hanari, MD, PhD, \\ Hisashi Gunji, MD, PhD, Takeshi Toyozumi, MD, PhD, Kentaro Murakami, MD, PhD, Masaya Uesato, MD, PhD, \\ Satoshi Ota, MD, PhD, and Hisahiro Matsubara, MD, PhD
}

Department of Frontier Surgery, Chiba University, Chiba, Japan

\begin{abstract}
Background. Postoperative docetaxel plus S-1 (DS) chemotherapy is expected to be the standard therapeutic strategy for pStage III gastric cancer based on the results of the JACCRO GC-07 study. Neoadjuvant chemotherapy (NAC) is thought to have several advantages over adjuvant settings.

Objective. This study aimed to compare the efficacies of NAC DS and the surgery-first strategy for advanced gastric cancer patients with D2 gastrectomy.

Methods. This was a retrospective, single-institution observational study. Of 171 patients with locally advanced (cStage IIB or III) gastric cancer who underwent curative D2 gastrectomy and received NAC DS and/or S-1 adjuvant chemotherapy between 2011 and 2017, 76 (after propensity score matching for 132 patients who met the eligibility criteria) were enrolled in this study. The 3 -year progression-free survival (PFS) rate was used to directly compare efficacies between NAC DS patients and surgery-first patients.

Results. The 3-year PFS rates for the NAC DS group were significantly higher than those for the surgery-first group (80.0 vs. 58.7; $p=0.037$ ), and the progression hazard ratio of the NAC DS group compared with the surgery-first
\end{abstract}

Electronic supplementary material The online version of this article (https://doi.org/10.1245/s10434-019-07299-7) contains supplementary material, which is available to authorized users.

(C) The Author(s) 2019

First Received: 13 October 2018;

Published Online: 11 April 2019

M. Kano, $\mathrm{MD}, \mathrm{PhD}$

e-mail: mkano@chiba-u.jp group was 0.394 (95\% confidence interval 0.159-0.978; $p=0.045)$.

Conclusions. The NAC DS group showed a high 3-year PFS compared with the surgery-first group, with standard S-1 postoperative chemotherapy or observation. NAC DS can be expected to be beneficial as the standard therapy for advanced gastric cancer and should be adopted for the test arm of a randomized controlled phase III trial.

Gastrectomy with D2 lymphadenectomy is a standard surgical procedure for locally advanced gastric cancer in Eastern Asia and is also recommended in Western countries, ${ }^{1,2}$ whereas surgery followed by adjuvant chemotherapy for locally advanced gastric cancer is the standard approach in Korea and Japan. ${ }^{3-5}$ Surgery plus adjuvant chemotherapy yields a 5-year survival rate of $70 \% ;^{6-8}$ however, patients' prognosis of a novel therapeutic strategy for locally advanced gastric cancer has been shown to have further reformability than standard D2 gastrectomy and adjuvant chemotherapy.

Neoadjuvant chemotherapy (NAC) has several advantages over adjuvant settings. For instance, it can eradicate systemic micrometastasis 9,10 and achieve downstaging to immediately start systemic treatment. In addition, the start of chemotherapy can be delayed if postoperative complications occur. Furthermore, postoperative compliance of chemotherapy is insufficient for patients who undergo gastrectomy. Indeed, 30-50\% of patients drop out of a 1-year course of chemotherapy after surgery. ${ }^{6,7}$

S-1 plus cisplatin (SC) is a standard treatment for metastatic gastric cancer in Eastern Asia. ${ }^{11}$ The Japan Clinical Oncology Group (JCOG) is currently conducting a phase III trial of NAC SC followed by surgery, and promising survival results have been reported from a small 
phase II trial evaluating two courses of NAC SC for bulky nodal disease. ${ }^{12}$ In addition, several phase II studies have demonstrated that NAC SC is safe and feasible. ${ }^{12-14}$ However, NAC SC phase III trials for large type 3 or 4 gastric cancers (JCOG0501) have not shown favorable results for NAC for advanced resectable gastric cancer. ${ }^{15}$

In Japan, docetaxel plus S-1 (DS) is thought to be an alternative regimen when the standard SC regimen cannot be used for metastatic gastric cancer. ${ }^{16}$ However, it has been suggested that DS may be superior to S-1 monotherapy with regard to survival in cases with nonmeasurable lesions. ${ }^{16}$ Paclitaxel and docetaxel are expected to be effective against peritoneal dissemination because of their high affinity for the peritoneum; ${ }^{; 1}$ therefore, this combination treatment may be a promising novel therapy for locally advanced gastric cancer.

In the present study, we evaluated the efficacy of NAC with DS for patients with advanced gastric cancer.

\section{PATIENTS AND METHODS}

We retrospectively investigated 171 patients who underwent curative D2 gastrectomy with or without S-1 adjuvant chemotherapy as the ACTS-GC (Adjuvant Chemotherapy Trial of TS-1 for Gastric Cancer) protocol for cStageIIB or III gastric cancer, between 2011 and 2017. The trial was conducted in accordance with the World Medical Association Declaration of Helsinki and the Japanese Ethical Guidelines for Medical and Health Research Involving Human Subjects. This protocol was approved by the Clinical Research Ethics Committee at Chiba University Hospital.

Patient medical records and survival status were retrospectively reviewed in August 2018. Patients were eligible for this study if they (1) had histologically proven advanced gastric cancer (including large type 3 or 4 , or bulky N) with clinical stage IIB or III disease (including T4b [liver, transverse colon, pancreatic body and tail, spleen and diaphragm) after radical gastrectomy with D2 lymph node dissection and R0 surgery; (2) were between 20 and 85 years of age; (3) had received no other treatments; (4) had been treated with S-1 chemotherapy within 8 weeks after surgery if they had (y)pStage II or III gastric cancer; (5) had more than 20 lymph nodes examined; and (6) had no synchronous or metachronous cancer. The data of 132 patients were examined after excluding 39 patients who did not meet these criteria retrospectively.

The 3-year progression-free survival (PFS) rate was determined to compare the efficacy of the NAC DS and surgery-first approaches. To reduce selection bias typically caused by the inclusion of imbalanced covariates in retrospective studies, the propensity score-matching method was used (Fig. 1). Tumor stage classification and D classification were in accordance with the Japanese Classification of Gastric Carcinoma (Third English Edition). ${ }^{18}$

\section{Chemotherapy Regimen}

Patients in the NAC DS group received two 21-day courses of NAC with the DS regimen $\left(\mathrm{S}-180 \mathrm{mg} / \mathrm{m}^{2}\right.$ from days 1 to 14 , and docetaxel $40 \mathrm{mg} / \mathrm{m}^{2}$ on day 1) and then underwent D2 gastrectomy, while patients in the surgeryfirst group received S-1 adjuvant chemotherapy if they had pStage II or III gastric cancer postoperatively, in accordance with the ACTS-GC protocol. ${ }^{6}$ It was intended that patients in the NAC DS group receive S-1 adjuvant chemotherapy.

\section{Follow-Up Evaluations}

For follow-up clinical studies, the patients' history and findings on physical examination, chest $\mathrm{x}$-ray, upper gastrointestinal endoscopy, and enhanced computed tomography $(\mathrm{eCT})$ of the abdomen and chest were performed at intervals of 3 months. When necessary, abdominal ultrasonic examinations, positron emission tomography (PET), and general bone scans were additionally examined.

\section{Statistical Analyses}

Clinicopathologic characteristics were summarized to provide a descriptive analysis, and mean \pm standard deviations were used to show quantitative variables of the NAC DS and surgery-first groups. Qualitative variables are expressed as frequency and percentage. A two-sample $t$ test for quantitative variables and Chi square test for qualitative variables were used to compare the characteristics of each group.

In non-randomized studies, effect estimates may be biased by group discordance; thus, propensity score-matched analysis was performed to minimize selection bias. Propensity scores were calculated using logistic regression. We included the following baseline covariates: age, sex, body mass index (BMI), Charlson Comorbidity Index, histology, tumor location, $\mathrm{cT}$ and $\mathrm{cN}$ factors, type of gastric resection, and type of surgical reconstruction. We purposefully used clinical $\mathrm{T}$ and $\mathrm{N}$ factors and variables collected before initial treatment as these represent the available data that physicians use to make decisions regarding stage prior to treatment. The confounding variables were input into the propensity score model. The nearest-neighbor matching method was applied, and one- 
FIG. 1 Enrolled patients. Thirty-nine patients were excluded according to the exclusion criteria. All 132 patients remaining after exclusion, along with 76 patients (39 in the NAC DS group and 37 in the surgery-first group) after propensity score matching, were analyzed. $N A C$ neoadjuvant chemotherapy, $D S$ docetaxel plus S-1

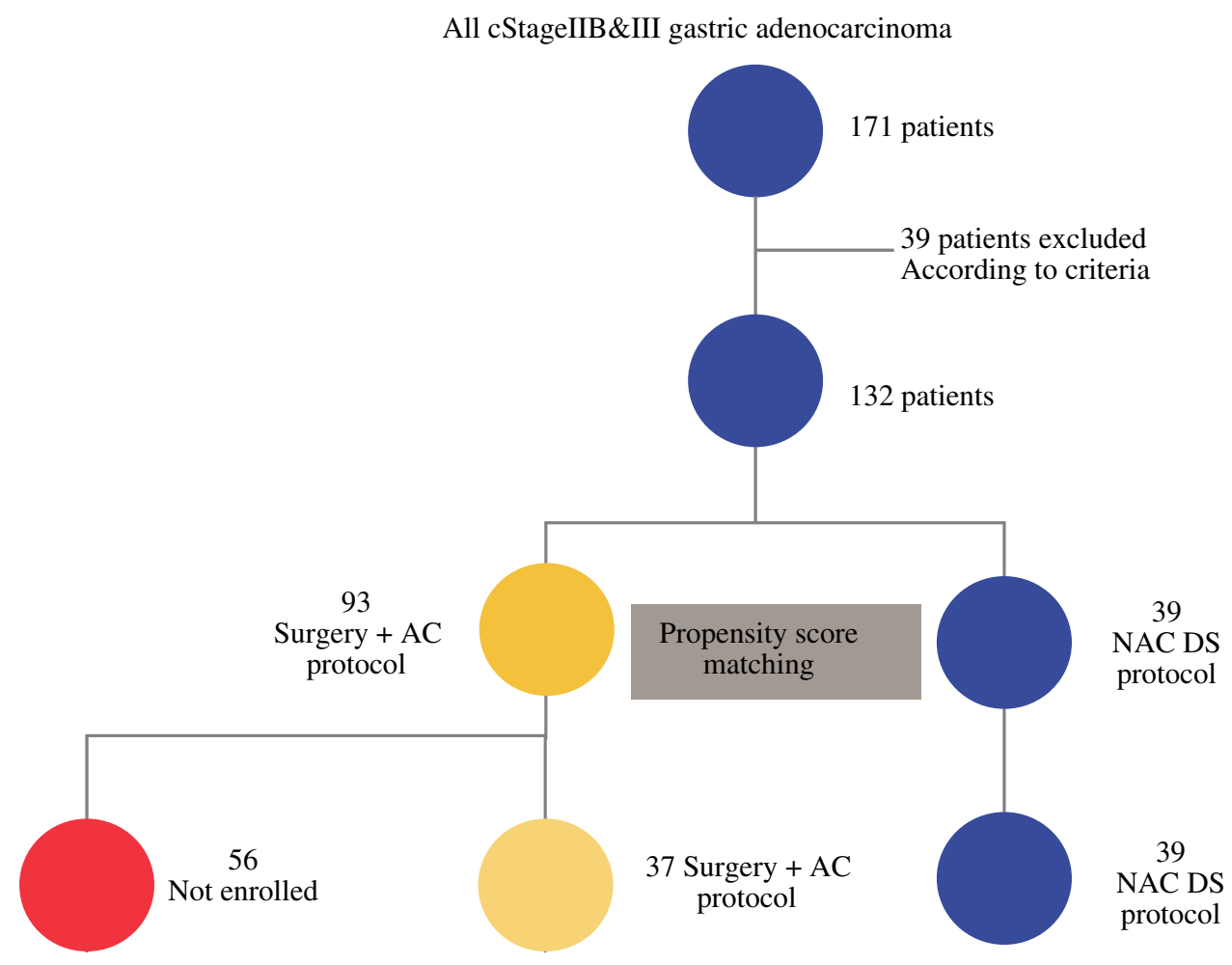

to-three matching between groups was achieved. The value of the caliper size was 0.25 .

The 3-year PFS rate was the primary endpoint of this study, and was defined as the period from the start of treatment to last follow-up, recurrence date, or death due to any cause, whichever came first. The 3-year PFS rate and 95\% confidence interval (CI) were calculated using the Kaplan-Meier method. The PFS rates between the NAC DS and surgery-first groups were compared using the logrank test. The Cox proportional hazards model was used to calculate the progression hazard ratio (HR) of the NAC DS group, compared with the surgery-first group as the reference, and the $95 \%$ CIs. All data analyses were performed by a medical statistician using the SPSS software package for Windows version 24.0 (IBM Corporation, Armonk, NY, USA). All tests were two-sided and statistical significance was set at $p<0.05$.

\section{Pathological Response Criteria of Neoadjuvant Chemotherapy (NAC)}

Pathological responses for gastric cancer after NAC were evaluated and graded by two pathologists, according to the proportion of necrosis in the tumor: grade 0 , no necrosis; grade 1a, less than one-third necrosis; grade $1 \mathrm{~b}$, more than one-third or less than two-thirds necrosis; grade 2 , less than two-thirds or more than all necrosis; and grade 3 , all parts of the tumors affected by necrosis. ${ }^{19}$

\section{RESULTS}

\section{Patient Characteristics}

Of the 132 patients eligible for this study, 39 received NAC DS and 93 received surgery first. The clinicopathologic characteristics of patients in the two groups are summarized in Table 1. Propensity score matching was used to compare the 3-year PFS rates between the two groups; after propensity score matching, 39 patients in the NAC DS group and 37 patients in the surgery-first group were matched. The differences in baseline characteristics between the two groups were statistically significant across the sex covariate before adjusting, however these differences were all eliminated without significant differences between the two groups after adjusting with propensity score matching (Table 1).

\section{Surgery and Postoperative Complications}

A total of 39 patients underwent D2 gastrectomy after NAC. The median operation time was 311 min (range 99-590), which was significantly longer than in the surgery-first group ( $p=0.006$, Mann-Whitney U-test), and the estimated blood loss was $645 \mathrm{~g}$ (range 5-2950), which was not markedly different from that in the surgery-first group $(p=0.086)$ after propensity score matching (Table 2$)$. 
TABLE 1 Demographics and clinicopathological characteristics in propensity score matched patients

\begin{tabular}{|c|c|c|c|c|c|c|}
\hline \multirow[t]{2}{*}{ Factors } & \multicolumn{3}{|l|}{ All patients } & \multicolumn{3}{|c|}{ Matched patients } \\
\hline & $\begin{array}{l}\text { NAC } \\
(N=39)\end{array}$ & $\begin{array}{l}\text { Surgery first } \\
(N=93)\end{array}$ & $P$ & $\begin{array}{l}\text { NAC } \\
(N=39)\end{array}$ & $\begin{array}{l}\text { Surgery first } \\
(N=37)\end{array}$ & $P$ \\
\hline Age & $69.3 \pm 7.76$ & $70.2 \pm 10.9$ & 0.378 & $69.3 \pm 7.76$ & $70.4 \pm 8.50$ & 0.557 \\
\hline \multicolumn{7}{|l|}{$\operatorname{Sex}, n(\%)$} \\
\hline Male & $32(82.1)$ & $59(62.8)$ & 0.0207 & $32(82.1)$ & $29(78.4)$ & 0.777 \\
\hline Female & 7 (17.9) & $37(39.4)$ & & $7(17.9)$ & $8(21.6)$ & \\
\hline BMI & $22.4 \pm 3.12$ & $22.4 \pm 3.44$ & 0.922 & $22.4 \pm 3.12$ & $22.2 \pm 3.13$ & 0.811 \\
\hline \multicolumn{7}{|c|}{ Charlson comorbidity index } \\
\hline 0 & $21(53.8)$ & $50(53.2)$ & 0.664 & $21(53.8)$ & $20(54.1)$ & 0.756 \\
\hline 1 & $11(28.2)$ & $20(21.3)$ & & $11(28.2)$ & $10(27.0)$ & \\
\hline 2 & $5(12.8)$ & $14(14.9)$ & & $5(12.8)$ & $3(8.1)$ & \\
\hline 3 & $2(5.1)$ & $10(10.6)$ & & $2(5.1)$ & $4(10.8)$ & \\
\hline \multicolumn{7}{|l|}{ Histology, $n(\%)$} \\
\hline Intestinal & $10(25.6)$ & $12(12.8)$ & 0.157 & $10(25.6)$ & $8(21.6)$ & 0.345 \\
\hline Diffuse & $7(17.9)$ & $25(26.6)$ & & $7(17.9)$ & $12(32.4)$ & \\
\hline Mixed & $22(56.4)$ & $57(60.6)$ & & $22(56.4)$ & $22(59.5)$ & \\
\hline \multicolumn{7}{|c|}{ Location of the tumor } \\
\hline $\mathrm{U}$ & $15(38.5)$ & $25(26.6)$ & 0.330 & $15(38.5)$ & $12(32.4)$ & 0.511 \\
\hline M & $9(23.1)$ & $31(33.0)$ & & $9(23.1)$ & $13(35.1)$ & \\
\hline $\mathrm{L}$ & $15(38.5)$ & $38(40.4)$ & & $15(38.5)$ & $12(32.4)$ & \\
\hline \multicolumn{7}{|l|}{$c T$ category, $n(\%)$} \\
\hline cT1 (M/SM) & $1(2.6)$ & $0(0)$ & 0.158 & $1(2.6)$ & $0(0)$ & 0.471 \\
\hline cT2 (MP) & $1(2.6)$ & $9(9.6)$ & & $1(2.6)$ & $3(8.1)$ & \\
\hline cT3 (SS) & $20(51.3)$ & $56(59.6)$ & & $20(51.3)$ & $19(51.4)$ & \\
\hline cT4a (SE) & $16(41.0)$ & $25(26.6)$ & & $16(41.0)$ & $12(32.4)$ & \\
\hline cT4b (SI) & $1(2.6)$ & $4(4.3)$ & & $1(2.6)$ & $3(8.1)$ & \\
\hline \multicolumn{7}{|l|}{$c N$ category, $n(\%)$} \\
\hline N0 & $5(12.8)$ & $6(3.6)$ & 0.220 & $5(12.8)$ & $2(5.4)$ & 0.264 \\
\hline N1-3 & $34(87.2)$ & $88(92.8)$ & & $34(87.2)$ & 35 (94.6) & \\
\hline \multicolumn{7}{|l|}{ cStage, $n(\%)$} \\
\hline cIIB & $17(43.6)$ & $27(28.7)$ & 0.0804 & $17(43.6)$ & $10(27.0)$ & 0.1316 \\
\hline cIIIA-C & $22(56.4)$ & $67(71.3)$ & & $22(56.4)$ & $27(73.0)$ & \\
\hline \multicolumn{7}{|c|}{ Radiographic type 4 or Large $(\geq 8 \mathrm{~cm})$ type 3} \\
\hline Yes & $11(28.2 \%)$ & $24(25.8 \%)$ & 0.7758 & $11(28.2 \%)$ & $10(27.2 \%)$ & 0.909 \\
\hline No & $28(71.8 \%)$ & $69(74.2 \%)$ & & $28(71.8 \%)$ & $27(72.8 \%)$ & \\
\hline \multicolumn{7}{|l|}{ Gastric resection } \\
\hline Distal Gastrectomy & $16(41.0)$ & $46(48.9)$ & 0.405 & $16(41.0)$ & $15(40.5)$ & 0.966 \\
\hline Total gastrectomy & $23(59.0)$ & $48(51.1)$ & & $23(59.0)$ & $22(59.5)$ & \\
\hline \multicolumn{7}{|l|}{ Reconstruction } \\
\hline Billroth I & $5(12.8)$ & $14(14.9)$ & 0.214 & $5(12.8)$ & $6(16.2)$ & 0.485 \\
\hline Billroth II & $1(2.6)$ & $11(11.7)$ & & $1(2.6)$ & $3(2.6)$ & \\
\hline Roux-en-Y & 33 (84.6) & 69 (73.4) & & 33 (84.6) & $28(75.7)$ & \\
\hline
\end{tabular}

$M$ mucosa/muscularis mucosa, $S M$ submucosa, $M P$ muscularis propria, $S S$ subserosa, $S E$ serosa, $S I$ invasion of adjacent structures
Postoperative complications were classified according to the Clavien-Dindo classification (Table 2). ${ }^{20}$ Complications of any grade in the NAC DS group occurred in nine patients $(23.1 \%)$, and the highest grade of complications was grade $3 \mathrm{a}$ in three patients $(7.7 \%)$. No patients died postoperatively. The rates of postoperative complications in the NAC DS group did not differ significantly from 
TABLE 2 Postoperative complications according to the Clavien-Dindo classification

\begin{tabular}{|c|c|c|c|c|c|c|}
\hline \multirow[t]{2}{*}{ Factors } & \multicolumn{3}{|l|}{ All patients } & \multicolumn{3}{|c|}{ Matched patients } \\
\hline & $\begin{array}{l}\text { NAC } \\
(N=39)\end{array}$ & $\begin{array}{l}\text { Surgery first } \\
(N=94)\end{array}$ & $P$ & $\begin{array}{l}\text { NAC } \\
(N=39)\end{array}$ & $\begin{array}{l}\text { Surgery first } \\
(N=37)\end{array}$ & $P$ \\
\hline \multicolumn{7}{|l|}{ Operative time (min) } \\
\hline Median (range) & $311(99-590)$ & $279(124-575)$ & 0.001 & $311(99-590)$ & $265(124-519)$ & 0.006 \\
\hline \multicolumn{7}{|l|}{ Intraoperative bleeding (g) } \\
\hline Median (range) & $645(5-2950)$ & $526(35-3700)$ & 0.001 & $645(5-2950)$ & $380(100-3700)$ & 0.086 \\
\hline \multicolumn{7}{|c|}{ Postoperative complications } \\
\hline Total $(\%)$ & $9(23.1)$ & $38(40.4)$ & 0.0567 & $9(23.1)$ & $15(40.5)$ & 0.101 \\
\hline Grade 1 & $4(10.3)$ & $17(18.1)$ & 0.260 & $4(10.3)$ & $2(5.4)$ & 0.433 \\
\hline Grade 2 & $2(5.1)$ & $11(11.7)$ & 0.245 & $2(5.1)$ & 7 (18.9) & 0.063 \\
\hline Grade 3 & $3(7.7)$ & $7(7.4)$ & 0.961 & $3(7.7)$ & $6(16.2)$ & 0.250 \\
\hline Grade 4 & $0(0.0)$ & $4(4.3)$ & 0.191 & $0(0.0)$ & $1(2.8)$ & 0.301 \\
\hline \multicolumn{7}{|c|}{ Complications of all grade, (\%) } \\
\hline Anastomotic leakage & $1(2.6)$ & $1(1.1)$ & 0.518 & $1(2.6)$ & $1(2.7)$ & 0.970 \\
\hline Pancreatic fistula & $6(15.4)$ & $9(9.6)$ & 0.335 & $5(15.4)$ & $5(13.5)$ & 0.929 \\
\hline Anastomotic stricture & $0(0.0)$ & $3(3.2)$ & 0.259 & $0(0.0)$ & $0(0.0)$ & \\
\hline Bowel obstruction & $0(0.0)$ & $5(5.3)$ & 0.142 & $0(0.0)$ & $2(5.4)$ & 0.141 \\
\hline Intraabodominal abscess & $0(0.0)$ & $2(2.1)$ & 0.359 & $0(0.0)$ & $1(2.7)$ & 0.301 \\
\hline Pneumonia & $0(0.0)$ & $6(6.4)$ & 0.106 & $0(0.0)$ & $3(8.1)$ & 0.067 \\
\hline Cholecystitis & $0(0.0)$ & $3(3.2)$ & 0.259 & $0(0.0)$ & $0(0.0)$ & 1.000 \\
\hline Others & $2(5.1)$ & $10(10.6)$ & 0.313 & $2(5.1)$ & $1(2.7)$ & 0.587 \\
\hline \multicolumn{7}{|c|}{ Complications of grade 3-4 } \\
\hline Pancreatic fistula & $3(7.7)$ & $6(6.4)$ & 0.784 & $3(7.7)$ & $2(5.4)$ & 0.643 \\
\hline Anastomotic stricture & $0(0.0)$ & $1(1.1)$ & 0.518 & $0(0.0)$ & $0(0.0)$ & 1.000 \\
\hline Bowel obstruction & $0(0.0)$ & $2(2.1)$ & 0.364 & $0(0.0)$ & $1(2.7)$ & 0.301 \\
\hline Pneumonia & $0(0.0)$ & $2(2.1)$ & 0.364 & $0(0.0)$ & $0(0.0)$ & 1.000 \\
\hline \multicolumn{7}{|c|}{ Postoperative hospital stay (days) } \\
\hline Median (range) & $14(9-57)$ & $15(7-106)$ & 0.397 & $14(9-57)$ & $14(10-70)$ & 0.695 \\
\hline
\end{tabular}

Gr, Grade; Clavien-Dindo Classification

${ }^{\mathrm{a}}$ The total does not add up because of overlapping elements

those in the surgery-first group after propensity score matching (Table 2).

\section{Pathological Findings and Downstaging after NAC}

Two patients achieved a pathological complete response after NAC DS (5.1\%). A pathological response of grade 0 , $1 \mathrm{a}, 1 \mathrm{~b}$, and 2 was observed in 5 (12.8\%), 12 (30.8\%), 9 (23.1\%), and $11(28.2 \%)$ patients, respectively. Data on the distribution of baseline $\mathrm{cT}, \mathrm{cN}$ and cStage (Table 1), and (y)pT, (y)pN and (y)pStage (Table 3) are shown. Although the baseline $\mathrm{cT}, \mathrm{cN}$, and cStage values were not significantly different between the two groups, the rate of ypT in the NAC DS group was significantly lower than the rate of pT in the surgery-first group.

\section{Progression-Free Survival}

The 3-year PFS rates were compared between the NAC DS and surgery-first groups (Table 4). The PFS rate in the NAC DS group was $80.0 \%$ (95\% CI 67.1-93.4\%), which was significantly higher $(p=0.037)$ than in the surgeryfirst group $(58.7 \%, 95 \%$ CI 41.9-75.4\%). A comparison between the NAC DS and surgery-first groups according to cStage (IIB or III) showed no marked differences between the two groups. For cStage IIB gastric cancer, the PFS rate for the NAC DS group $(80.2 \%, 95 \%$ CI $60.0-100.4 \%)$ did not differ significantly from that in the surgery-first group $(70.0 \%, 95 \%$ CI $41.6-98.4 \% ; p=0.504)$. Furthermore, no marked differences in the PFS rates for cStage III (IIIA, IIIB, and IIIC) patients were noted between the NAC DS $(80.0 \%, 95 \%$ CI $62.5-97.5 \%)$ and surgery-first groups 
TABLE 3 Pathological findings in resected patients

\begin{tabular}{|c|c|c|c|c|c|c|}
\hline \multirow[t]{2}{*}{ Factors } & \multicolumn{3}{|c|}{ All patients } & \multicolumn{3}{|c|}{ Matched patients } \\
\hline & $\begin{array}{l}\text { NAC } \\
(N=39)\end{array}$ & $\begin{array}{l}\text { Surgery first } \\
(N=94)\end{array}$ & $P$ & $\begin{array}{l}\text { NAC } \\
(N=39)\end{array}$ & $\begin{array}{l}\text { Surgery first } \\
(N=37)\end{array}$ & $P$ \\
\hline \multicolumn{7}{|c|}{$p T$ category, $n(\%)$} \\
\hline pT0 & $2(5.1)$ & $0(0)$ & 0.0002 & $2(5.1)$ & $0(0)$ & 0.027 \\
\hline $\mathrm{pT} 1(\mathrm{M} / \mathrm{SM})$ & $5(12.9)$ & $7(7.4)$ & & $5(12.9)$ & $1(2.7)$ & \\
\hline pT2(MP) & $12(30.8)$ & $7(7.4)$ & & $12(30.8)$ & $5(13.5)$ & \\
\hline pT3(SS) & $13(33.3)$ & $32(34.0)$ & & $13(33.3)$ & $12(32.4)$ & \\
\hline pT4a(SE) & $7(17.9)$ & $39(41.5)$ & & $7(17.9)$ & $12(32.4)$ & \\
\hline pT4b(SI) & $0(0)$ & $9(9.6)$ & & $0(0)$ & $4(10.8)$ & \\
\hline \multicolumn{7}{|c|}{$p N$ category, $n(\%)$} \\
\hline pNO & $15(38.5)$ & $13(13.8)$ & 0.0126 & $15(38.5)$ & $6(16.2)$ & 0.1466 \\
\hline pN1 & $8(20.5)$ & $25(26.6)$ & & $8(20.5)$ & $11(29.7)$ & \\
\hline $\mathrm{pN} 2$ & $9(23.1)$ & $31(33.0)$ & & $9(23.1)$ & $10(27.0)$ & \\
\hline $\mathrm{pN} 3$ & $6(15.4)$ & $25(26.6)$ & & $6(15.4)$ & $10(27.0)$ & \\
\hline \multicolumn{7}{|c|}{ pStage, $n(\%)$} \\
\hline pTONO & $2(5.1)$ & $0(0.0)$ & 0.001 & $2(5.1)$ & $0(0.0)$ & 0.0966 \\
\hline pIA-B & $10(25.6)$ & $8(8.5)$ & & $10(25.6)$ & $6(16.2)$ & \\
\hline pIIA-B & $13(33.3)$ & $23(24.5)$ & & $13(33.3)$ & 8 (21.6) & \\
\hline pIIIA-C & $14(35.9)$ & $63(67.0)$ & & $14(35.9)$ & $23(62.2)$ & \\
\hline
\end{tabular}

TABLE 4 Disease-free survival rate for the NAC DS and Surgery first groups according to stages (Japanese Classification of Gastric Carcinoma (Third English Edition) using the log-rank test

\begin{tabular}{llll}
\hline Stages & $\begin{array}{l}\text { NAC DS } \\
(n=39)\end{array}$ & $\begin{array}{l}\text { Surgery first } \\
(n=37)\end{array}$ & $p$ value \\
\hline All & $80.0(67.1-93.4)$ & $58.7(41.9-75.4)$ & 0.037 \\
cStage IIB & $80.2(60.0-100.4)$ & $70.0(41.6-98.4)$ & 0.504 \\
cStage III & $80.0(62.5-97.5)$ & $54.0(33.6-74.4)$ & 0.054 \\
\hline Pathological response & $\begin{array}{l}\text { NAC DS } \\
(n=39)\end{array}$ & $\begin{array}{l}\text { Surgery first } \\
(n=37)\end{array}$ & $p$ value \\
\hline Grade 1a $\geqq$ & $76.4(56.3-96.6)$ & $58.7(41.9-75.4)$ & 0.321 \\
Grade 1b & $90.5(77.9-103.0)$ & & 0.020 \\
\hline
\end{tabular}

(54.0\%, 95\% CI 33.6-74.4; $p=0.054)$. The PFS graphs of patients with cStage IIB and/or III gastric cancer are shown in Fig. 2a.

A comparison of the NAC DS and surgery-first groups according to postoperative pathological responses (grade $\leq 1 \mathrm{a}$ or $\geq 1 \mathrm{~b}$ ) showed differences in grade $\geq 1 \mathrm{~b}$ responses between the two groups. The PFS rate for cases with a grade $\leq 1$ a pathological response after NAC DS and surgery $(76.4 \%$, 95\% CI $56.3-96.3 \%)$ did not differ significantly from that in the surgery-first group $(p=0.321)$. Additionally, the PFS rate for cases with a grade $\geq 1 \mathrm{~b}$ pathological response after NAC DS and surgery $(90.5 \%, 95 \%$ CI $77.9-103.0)$ was higher than in the surgery-first group ( $p=0.020)$. The PFS graphs of patients with grade $\leq 1 \mathrm{a}$ or $\geq 1 \mathrm{~b}$ pathological responses after NAC DS compared with the surgery-first group are shown in Fig. $2 b$.

\section{A Comparison of the Hazard Ratios for Recurrence Between the NAC DS and Surgery-First Groups}

The HR for progression of the NAC DS group was calculated using the Cox proportional hazard model to compare surgery first as the reference (Table 5). The HR of the NAC DS group for all patients after propensity score matching was 0.394 (95\% CI 0.159-0.978; $p=0.045$ ). This study could not show that NAC DS was significantly related to a decreased risk of recurrence for patients with stage IIB (HR $0.583,95 \%$ CI $0.118-2.892 ; p=0.509$ ) or stage III disease (HR 0.344. 95\% CI 0.109-1.083; $p=0.068)$. The HR of gastric cancer progression in cases with grade $\geq 1 \mathrm{~b}$ postoperative pathological responses compared with surgery first as the reference was 0.207 (95\% CI $0.047-0.912 ; p=0.037$ ). Furthermore, this study did not find NAC DS to be significantly associated with a decreased risk of gastric cancer recurrence for patients with grade $\geq 1$ a pathological responses (HR 0.602, 95\% CI $0.216-1.679 ; p=0.330$ ). 
(a) cStage IIB\&III

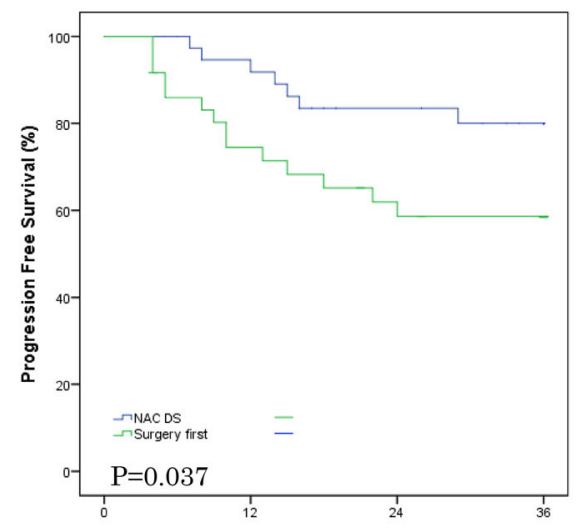

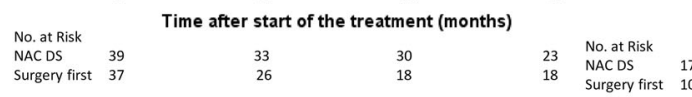

(d) Grade $\leq 1 \mathrm{a}$

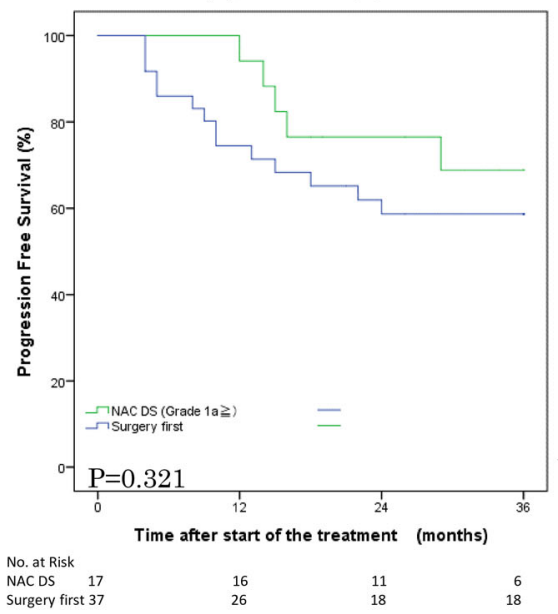

FIG. 2 (a-c) Progression-free survival of patients with cStage IIB and III, cStage IIB, and cStage III gastric cancer using the KaplanMeier method. (d, e) Progression-free survival of patients with grade

TABLE 5 Hazard ratio of NAC DS for recurrence of gastric cancer compared with surgery first as a reference

\begin{tabular}{lll}
\hline Stages & Hazard ratio $(95 \%$ CI $)$ & $p$ value \\
\hline All & $0.394(0.159-0.978)$ & 0.045 \\
cStage IIB & $0.583(0.118-2.892)$ & 0.509 \\
cStage III & $0.344(0.109-1.083)$ & 0.068 \\
Grade 1a $\geqq$ & $0.602(0.216-1.672)$ & 0.330 \\
Grade 1b $\leqq$ & $0.207(0.047-0.912)$ & 0.037 \\
\hline
\end{tabular}

(b) cStage IIB

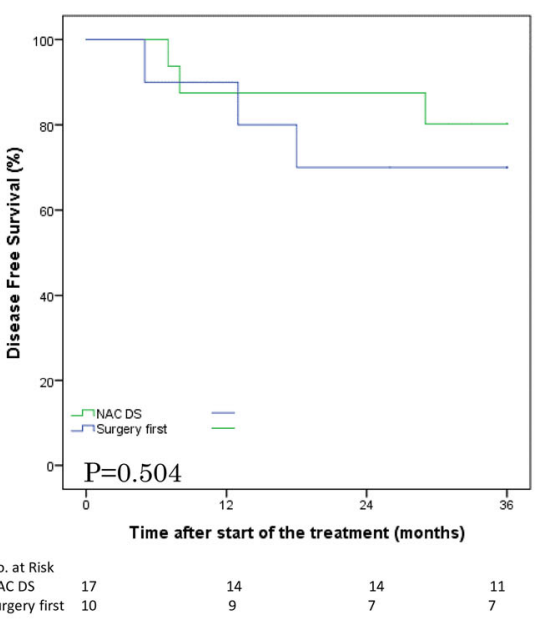

(c) cStage III

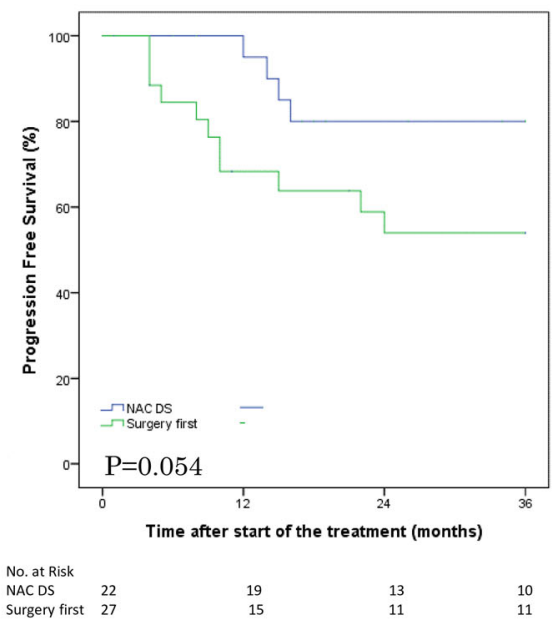

(e) Grade $\geq 1 b$

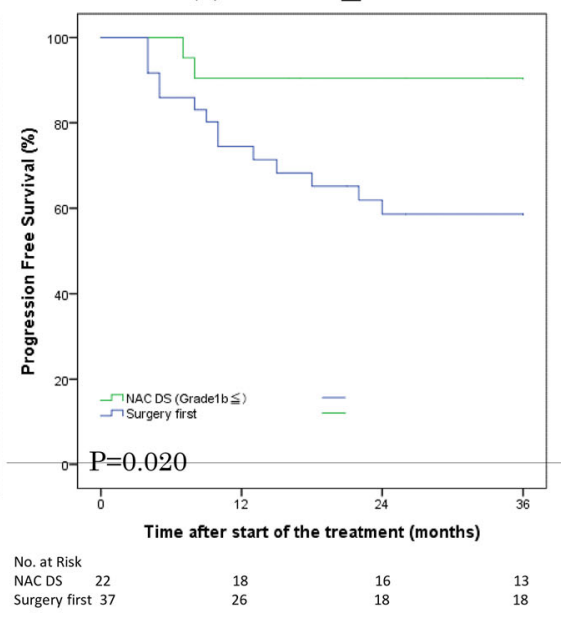

$\leq 1 \mathrm{a}$ and grade $\geq 1 \mathrm{~b}$ pathological responses after NAC DS compared with the surgery-first group using the Kaplan-Meier method. NAC neoadjuvant chemotherapy, $D S$ docetaxel plus S-1

\section{DISCUSSION}

The effectiveness of DS adjuvant chemotherapy for pStage III gastric cancer was recently reported (JACCRO GC-07) at the 2018 American Society of Clinical Oncology annual meeting. ${ }^{5}$ The DS protocol of this study, in which its safety and efficacy as adjuvant chemotherapy for pStage III gastric cancer in a phase III RCT (JACCRO GC-07) was proven.

We completed a phase II trial for evaluation of the safety of NAC DS for patients with locally advanced gastric cancer followed by standard D2 gastrectomy. In the phase II trial (in submission), the authors judged NAC DS and surgery to be acceptable for daily clinical use based on the completion rate of NAC and surgery, R0 resection rate, adverse events of chemotherapy, and surgery. Staging 
laparoscopy was required in all cases prior to NAC treatment for advanced gastric cancer patients, to obtain a precise diagnosis of cancer staging.

We selected clinical stage IIB and III gastric cancer patients for this study because the 5-year survival rate for clinical stage IIB gastric cancer is $<70 \%$, therefore a more effective treatment than D2 gastrectomy with adjuvant chemotherapy is needed in this field.

Our present study showed that the 3-year PFS rates in the NAC DS group for cStage IIB and III gastric cancer patients were higher than those in the surgery-first group. Furthermore, the HR of progression in the NAC DS group, compared with the surgery-first group, was 0.394 .

This study had some limitations as it was a retrospective study and only early outcomes were observed. Although the 3-year PFS is meaningful because most gastric cancer recurrences are known to occur within 3 years, ${ }^{21}$ an expanded or well-designed prospective RCT is warranted in order to confirm our results.

Nevertheless, the present results suggest that NAC DS chemotherapy should be considered for the test arm of an RCT, compared with standard treatment. Only patients from a single institution were enrolled in this study; therefore, an international study with standardized D2 dissection may lead to more valuable research results.

Whether doublet or triplet regimens should be used for NAC is controversial. Satisfactory 3-year overall survival rates of perioperative triplet regimens in a phase II trial have been reported, ${ }^{22-24}$ and reports on perioperative chemotherapy using triplet regimens have shown a high rate of grade 3/4 adverse events. Furthermore, phase III trials of docetaxel and cisplatin combination therapy for metastatic gastric cancer (JCOG1013) have not shown favorable results. ${ }^{25}$

Relatively large differences among pathological responses in the 3-year PFS and HR of the risk of gastric cancer progression were observed in this study, suggesting that the development of a companion diagnosis for NAC is important.

Treatment approaches using molecular-targeted drugs and immune checkpoint inhibitors for metastatic gastric cancer treatment are currently being developed, ${ }^{26-29}$ and are promising from the point of view of precision oncology. ${ }^{30-32}$ In the nearest future, molecular-targeted drugs and immune checkpoint inhibitors will appear in the field of perioperative chemotherapy development.

\section{CONCLUSIONS}

NAC DS with D2 gastrectomy had a higher PFS rate than the surgery-first approach for patients with cStage IIB and III gastric cancer. In addition, surgery first was found to be associated with more than twice the risk of progression as NAC DS. Although previous studies have not confirmed the effectiveness of NAC for advanced gastric cancer, NAC DS should be considered preferentially for the test arm of an RCT for patients with locally advanced gastric cancer.

OPEN ACCESS This article is distributed under the terms of the Creative Commons Attribution 4.0 International License (http://crea tivecommons.org/licenses/by/4.0/), which permits unrestricted use, distribution, and reproduction in any medium, provided you give appropriate credit to the original author(s) and the source, provide a link to the Creative Commons license, and indicate if changes were made.

\section{REFERENCES}

1. Cunningham D, Allum WH, Stenning SP, et al. Perioperative chemotherapy versus surgery alone for resectable gastroesophageal cancer. The N Engl J Med. 2006;355(1):11-20.

2. Ychou M, Boige V, Pignon JP, et al. Perioperative chemotherapy compared with surgery alone for resectable gastroesophageal adenocarcinoma: an FNCLCC and FFCD multicenter phase III trial. J Clin Oncol. 2011;29(13):1715-21.

3. Japanese gastric cancer treatment guidelines 2010 (ver. 3). Gastric Cancer. 2011;14(2):113-23.

4. Lee JH, Kim JG, Jung HK, et al. Clinical practice guidelines for gastric cancer in Korea: an evidence-based approach. J Gastric Cancer. 2014;14(2):87-104.

5. Kodera Y, Yoshida K, Kochi M, et al. A randomized phase III study comparing S-1 plus docetaxel with S-1 alone as a postoperative adjuvant chemotherapy for curatively resected stage III gastric cancer (JACCRO GC-07 trial). J Clin Oncol. 2018;36(15 Suppl):4007.

6. Sakuramoto S, Sasako M, Yamaguchi T, et al. Adjuvant chemotherapy for gastric cancer with $\mathrm{S}-1$, an oral fluoropyrimidine. The N Engl J Med. 2007;357(18):1810-20.

7. Bang YJ, Kim YW, Yang HK, et al. Adjuvant capecitabine and oxaliplatin for gastric cancer after D2 gastrectomy (CLASSIC): a phase 3 open-label, randomised controlled trial. Lancet. 2012;379(9813):315-21.

8. Sasako M, Sakuramoto S, Katai H, et al. Five-year outcomes of a randomized phase III trial comparing adjuvant chemotherapy with S-1 versus surgery alone in stage II or III gastric cancer. J Clin Oncol. 2011;29(33):4387-93.

9. Chung HW, Jang S, Kim H, et al. Combined targeting of highmobility group box-1 and interleukin- 8 to control micrometastasis potential in gastric cancer. Int $\mathrm{J}$ Cancer. 2015;137(7):1598-609.

10. Zhang ZY, Ge HY. Micrometastasis in gastric cancer. Cancer Lett. 2013;336(1):34-45.

11. Koizumi W, Narahara H, Hara T, et al. S-1 plus cisplatin versus S-1 alone for first-line treatment of advanced gastric cancer (SPIRITS trial): a phase III trial. The Lancet Oncol. 2008;9(3):215-21.

12. Yoshikawa T, Sasako M, Yamamoto S, et al. Phase II study of neoadjuvant chemotherapy and extended surgery for locally advanced gastric cancer. The Br J Surg.. 2009;96(9):1015-22.

13. Kochi M, Fujii M, Kanamori N, et al. Phase II Study of Neoadjuvant Chemotherapy With S-1 and CDDP in Patients With Lymph Node Metastatic Stage II or III Gastric Cancer. Am J Clin Oncol. 2017;40(1):17-21. 
14. Yoshikawa T, Taguri M, Sakuramoto S, et al. A comparison of multimodality treatment: two and four courses of neoadjuvant chemotherapy using S-1/CDDP or S-1/CDDP/docetaxel followed by surgery and $\mathrm{S}-1$ adjuvant chemotherapy for macroscopically resectable serosa-positive gastric cancer: a randomized phase II trial (COMPASS-D trial). Jpn J Clin Oncol. 2012;42(1):74-7.

15. Iwasaki $Y$, Terashima M, Mizusawa J, et al. Randomized phase III trial of gastrectomy with or without neoadjuvant S-1 plus cisplatin for type 4 or large type 3 gastric cancer: Japan Clinical Oncology Group study (JCOG0501). J Clin Oncol. 2018;36(15 Suppl):4046.

16. Koizumi W, Kim YH, Fujii M, et al. Addition of docetaxel to S-1 without platinum prolongs survival of patients with advanced gastric cancer: a randomized study (START). J Cancer Res Clin Oncol. 2014;140(2):319-28.

17. Kobayashi M, Sakamoto J, Namikawa T, et al. Pharmacokinetic study of paclitaxel in malignant ascites from advanced gastric cancer patients. World J Gastroenterol. 2006;12(9):1412-5.

18. High TMB Predicts Immunotherapy Benefit. Cancer Discov. 2018;8(6):668.

19. Kim IH, Park SS, Lee CM, et al. Efficacy of adjuvant S-1 versus XELOX chemotherapy for patients with gastric cancer after D2 lymph node dissection: a retrospective, multi-center observational study. Ann Surg Oncol. 2018;25(5):1176-83.

20. Dindo D, Demartines N, Clavien PA. Classification of surgical complications: a new proposal with evaluation in a cohort of 6336 patients and results of a survey. Ann Surg 2004;240(2): 205-13.

21. Japanese gastric cancer treatment guidelines 2014 (ver. 4). Gastric cancer: official journal of the International Gastric Cancer Association and the Japanese Gastric Cancer Association. 2017;20(1):1-19.

22. Oki E, Emi Y, Kusumoto T, et al. Phase II study of docetaxel and S-1 (DS) as neoadjuvant chemotherapy for clinical stage III resectable gastric cancer. Ann Surg Oncol. 2014;21(7):2340-6.

23. Fujitani K, Tamura S, Kimura Y, et al. Three-year outcomes of a phase II study of adjuvant chemotherapy with S-1 plus docetaxel for stage III gastric cancer after curative D2 gastrectomy. Gastric Cancer. 2014;17(2):348-53.

24. Hirakawa M, Sato Y, Ohnuma H, et al. A phase II study of neoadjuvant combination chemotherapy with docetaxel, cisplatin, and S-1 for locally advanced resectable gastric cancer: nucleotide excision repair (NER) as potential chemoresistance marker. Cancer Chemother Pharmacol. 2013;71(3):789-97.

25. Yamada Y, Boku N, Mizusawa J, et al. Phase III study comparing triplet chemotherapy with S-1 and cisplatin plus docetaxel versus doublet chemotherapy with S-1 and cisplatin for advanced gastric cancer (JCOG1013). J Clin Oncol. 2018;36(15 Suppl):4009.

26. Wilke H, Muro K, Van Cutsem E, et al. Ramucirumab plus paclitaxel versus placebo plus paclitaxel in patients with previously treated advanced gastric or gastro-oesophageal junction adenocarcinoma (RAINBOW): a double-blind, randomised phase 3 trial. The Lancet Oncol. 2014;15(11):1224-35.

27. Tran PN, Sarkissian S, Chao J, et al. PD-1 and PD-L1 as emerging therapeutic targets in gastric cancer: current evidence. Gastrointest Cancer. 2017;7:1-11.

28. Bang YJ, Cho JY, Kim YH, et al. Efficacy of sequential ipilimumab monotherapy vs best supportive care for unresectable locally advanced/metastatic gastric or gastroesophageal junction cancer. Clin Cancer Res. 2017.

29. Kang YK, Boku N, Satoh T, et al. Nivolumab in patients with advanced gastric or gastro-oesophageal junction cancer refractory to, or intolerant of, at least two previous chemotherapy regimens (ONO-4538-12, ATTRACTION-2): a randomised, double-blind, placebo-controlled, phase 3 trial. Lancet. 2017;390(10111): 2461-71.

30. Li X, Pasche B, Zhang W, et al. Association of MUC16 Mutation With Tumor Mutation Load and Outcomes in Patients With Gastric Cancer. JAMA Oncol. 2018;4(12):1691-1698.

31. Lianos GD, Glantzounis GK, Bali CD, et al. Identification of novel genes by whole-exome sequencing can improve gastric cancer precision oncology. Future Oncol. 2017;13(10):883-92.

32. Pietrantonio F, Fuca G, Morano F, et al. Biomarkers of primary resistance to trastuzumab in HER2-positive metastatic gastric cancer patients: the AMNESIA case-control study. Clin Cancer Res. 2018;24(5):1082-9.

Publisher's Note Springer Nature remains neutral with regard to jurisdictional claims in published maps and institutional affiliations. 